\title{
Scaling of the Beam Plasma Discharge for Low Magnetic Fields
}

\author{
K. Papadopoulos
}

\author{
Department of Physics and Astronomy, University of Maryland, College Park
}

\begin{abstract}
A theoretical analysis of the scaling law and the value of the threshold current for beam plasma discharge (BPD) is presented, based on the requirement for an absolute instability near the plasma frequency. It is shown that both the scaling law as well as the numerical values of $I_{c}$ are consistent with the experimental data, in the low pressure regimes and for weak magnetic field experiments $\left(\omega_{e}>\Omega_{e}\right)$ if the dominant particle loss mechanism is due to Bohm diffusion. The implications of the findings to electron injection in space are discussed.
\end{abstract}

\section{INTRODUCTION}

Laboratory studies of energetic electron beam injection in a neutral gas filled vacuum chamber carried out in the large vacuum facility at the Johnson Space Center (JSC) [Bernstein et al., $19 \overline{9} 78,1979,1980]$ have provided important informations for the interpretation of data from space based electron beam injection. Perhaps the most important aspect was the determination of an empirical relationship of the form [Kellogg et al., 1982]

$$
I_{c} \sim \frac{E_{b}^{3 / 2}}{B^{\lambda} L} f(p)
$$

for the critical current $I_{c}$ required for beam plasma discharge ignition in terms of the values of beam energy $\left(E_{b}\right)$, ambient magnetic field $(B)$, system length $L$, and ambient pressure $p$. The relationship is most commonly known in its low pressure form,

$$
I_{c} \sim \frac{E_{b}{ }^{3 / 2}}{B^{\lambda} p^{\mu} L}
$$

which for the large vacuum chamber was valid for pressures $p<p_{0}=20 \mu \mathrm{T}$. The best fit to the data gave values of $\lambda \approx 0.5-1$ and $\mu \approx 0.5-1$. For pressures above $20 \mu \mathrm{T}$ the dependence of $I_{c}$ on pressure took the form $I_{c} \sim p^{\mu}$. Most of the data were obtained in the low pressure regime and a systematic study of the high pressure regime has yet not been performed. Similar results have been reproduced in a number of other experiments [Konradi et al., 1983; Lyakhov et al., 1982; Bernstein et al., 1983; Kawashima et al., 1983] in which the collisional ionization by the beam is sufficient to bring the plasma density of the system to the point that $\omega_{e}>\Omega_{e}$, where $\omega_{e}, \Omega_{e}$ are the plasma and cyclotron frequency.

Triggering of BPD has been long associated with a beam plasma instability between the electron beam and the beam generated plasma [Kharchenko et al., 1962; Getty and Smullin, 1963; Galeev et al., 1976, 1983; Linson and Papadopoulos, 1980; Papadopoulos, 1982]. For $\omega_{e}>\Omega_{e}$ and finite size systems Rowland et al. [1981] and Papadopoulos [1982], have associated the triggering of the BPD with the threshold for an absolute beam plasma instability near $\omega_{e}$. As explained in Papadopoulos [1982] for systems such as the JSC tank the system length $(L \$ 20 \mathrm{~m}$ ) is not long enough to allow convective modes to grow to sufficient amplitude. The requirement that the waves grow at frequencies near $\omega_{e}$ is connected with the fast rate of nonlinear energy transfer of the beam energy to

Copyright 1986 by the American Geophysical Union.

Paper number 5A0840.

$0148-0227 / 86 / 005 \mathrm{~A}-0840 \$ 05.00$ ionizing suprathermal electron tails [Papadopoulos and Coffey, 1974; Papadopoulos, 1975; Papadopoulos and Rowland, 1978; Rowland et al., 1981; Galeev et al., 1983]. It is the purpose of this paper to develop a model for BPD ignition at low pressures and the expected scaling laws on the basis of the criterion for an absolute instability near $\omega_{e}$. Notice that for systems with $\omega_{e}<\Omega_{e}$ the $\omega_{e}$ waves are in the lower hybrid branch [Manickam et al., 1975] in which case, in the absence of internal wave reflections, they always give convective amplification. Our analysis therefore applies only to situations where $\omega_{e}>\Omega_{e}$.

The plan of the paper is as follows. We discuss next the beam plasma equilibrium expected on the basis of collisional ionization. Section 3 presents the instability theory for the configuration determined in section 2 and derives the threshold criteria. Section 4 presents a comparison of the model with the BPD ignition values determined in the JSC experiment. The final section summarizes the findings and discusses their applicability to other situations. The numerical values are given in MKS units except for the beam energy (keV), pressure $(\mu \mathrm{T})$, magnetic field $(G)$, and density $\left(\mathrm{cm}^{-3}\right)$.

\section{Pre-BPD Density BuilduP}

Before entering the instability analysis it is necessary to establish the equilibrium density profiles for the plasma during the collisional ionization stage. We assume that the beam density profile is given by

$$
n_{b}(r)=n_{b} e^{-r^{2} / a^{2}}=\frac{I_{b}}{e V_{b} \pi a^{2}} e^{-r^{2} / a^{2}}
$$

where $I_{b}$ and $V_{b}$ are the electron beam current and velocity parallel to the magnetic field and $a$ the beam radius given by

$$
a=\frac{V_{b} \sin \theta_{d}}{\Omega_{e}}
$$

where $\theta_{d}$ is the effective divergence injection angle. The effective divergence angle $\theta_{d}$ has been defined so that it includes the beam spreading caused by the initial space charge neutralization processes [Linson and Papadopoulos, 1980]. The equation for the ionization at midplane (i.e., ignoring the $z$ dependence) is

$$
\frac{\partial}{\partial t} n(r)-\frac{1}{r} \frac{\partial}{\partial r} r D \frac{\partial}{\partial r} n(r)+\frac{\alpha n(r)}{L}=\frac{I_{b} N_{0} \sigma}{e \pi a^{2}} e^{-r^{2} / a^{2}}
$$

where $D$ is the diffusion coefficient, $L$ the system length, $N_{0}$ the ambient neutral density, and $\sigma$ the ionization cross section. The term $(\alpha / L) n(r)$ describes the axial losses. Ignoring the $z$ dependence in both the beam and the plasma can be easily justified for the pre-BPD stage of the laboratory experiments since the mean free path $\lambda$ for energy deposition is much 
longer than the system size. The equivalent condition for space experiments will be discussed in our concluding remarks. It is worth noting that if we average (2) over the volume we recover the zero dimensional description in terms of the confinement time $\tau$ [Papadopoulos, 1982], i.e.,

$$
\frac{d}{d t} n=\frac{I_{b}}{e \pi a^{2}} N_{0} \sigma-\frac{n}{\tau}
$$

which gives the steady state value of the density as

$$
n=\frac{I_{b}}{e \pi a^{2}} N_{0} \sigma \tau
$$

The general solution of (2) in terms of the first and second order Bessel functions $I_{0}, K_{0}$ is

$$
n(r)=A_{1} I_{0}\left(\frac{r}{b}\right)+A_{2} K_{0}\left(\frac{r}{b}\right)
$$

with

$$
b^{2}=\frac{L D}{\alpha^{2}}
$$

The constants $A_{1}, A_{2}$ are determined from the boundary conditions. In the thin beam limit $(a \ll b)$, in which we have a line source, we find

$$
n(r)=\frac{I_{b} N_{0} \sigma}{2 \pi e D} K_{0}\left(\frac{r}{b}\right) \equiv n_{0} K_{0}\left(\frac{r}{b}\right)
$$

The more general solution gives, for regions inside the source $(0 \leq r \leq a)$

$$
n(r)=\frac{I_{b} N_{0} \sigma}{e D \pi a^{2}} \int_{0}^{\infty} K_{0}\left(\frac{\left|r-r^{\prime}\right|}{b}\right) \exp \left(-r^{\prime 2} / a^{2}\right) d r^{\prime}
$$

while outside the source $(a \leq r \leq b)$

$$
n(r)=\frac{I_{b} N_{0} \sigma}{e D \pi a^{2}}\left(\int_{0}^{\infty} I_{0}\left(\frac{r^{\prime}}{b}\right) \exp \left(-r^{\prime 2} / a^{2}\right) r^{\prime} d r^{\prime}\right) K_{0}\left(\frac{r}{b}\right)
$$

Guided by the experimental results we restrict ourselves here to the thin beam limit $a<b$, which implies that $\left(V_{b} \sin \right.$ $\left.\theta_{d}\right) / \Omega_{e}<\left(D^{1 / 2} L^{1 / 2}\right) / \alpha$. In this case the input parameters required for the instability analysis are $n_{b}, n_{0}, a$, and $b$ and are given by (1), (6) and (7). The values of $n_{b}$ and $n_{0}$ in the system units discussed in section 1 can be found from (1) and (7) as

$$
\begin{gathered}
n_{b}=1.9 \times 10^{6} \frac{I_{b} B^{2}}{E_{b}^{3 / 2}} \frac{1}{\sin ^{2} \theta_{d}} \\
n_{0}=3.2 \times 10^{8} \frac{I_{b} P}{E_{b}^{1 / 2}} \frac{1}{D}
\end{gathered}
$$

\section{INSTABILITY THEORY}

The homogeneous interaction between the beam and the plasma is described by the dispersion relation

$$
\varepsilon(k, \omega)=1+K_{p}(k, \omega)+K_{b}(k, \omega)=0
$$

where $K_{p}$ and $K_{b}$ are the longitudinal dielectric functions of the plasma and the beam. Both $K_{p}$ and $K_{b}$ can be calculated for any type of distribution functions including collisional and finite size geometry effects [Briggs, 1964]. We choose here models that allow us to emphasize the physics and avoid the mathematical complexity. Consistently with Rowland et al. [1981] and Szuszczewicz et al. [1982], we consider only the synchronous Cerenkov interaction of a slow beam wave with an upper hybrid wave $\omega_{0}$ of the cold plasma. In this case

$$
\begin{gathered}
K_{p}(\mathbf{k}, \omega)=-\frac{\omega_{0}{ }^{2}}{\omega^{2}} \\
\omega_{0}{ }^{2}=\frac{1}{2}\left(\omega_{e}{ }^{2}+\Omega_{e}{ }^{2}\right)+\left[\frac{1}{4}\left(\omega_{e}{ }^{2}+\Omega_{e}{ }^{2}\right)^{2}-\omega_{e}{ }^{2} \Omega_{e}{ }^{2} \cos ^{2} \theta\right]^{1 / 2} \\
K_{b}(k, \omega)=-\frac{\omega_{b}{ }^{2} R \cos ^{2} \theta}{\left(\omega-k_{z} V_{b}\right)^{2}} \\
\cos ^{2} \theta=\frac{k_{z}{ }^{2}}{k_{z}{ }^{2}+k_{\perp}{ }^{2}}
\end{gathered}
$$

$\omega_{e}, \omega_{b}$ and $\Omega_{e}$ are the plasma frequency, beam plasma frequency, and cyclotron frequency respectively, and $k^{2}=k_{z}{ }^{2}$ $+k_{\perp}^{2}$. The potential was assumed to have the form $J_{0}\left(k_{\perp} r\right)$ $\exp \left[i\left(k_{z} z-\omega t\right)\right]$ and thus the propagation is axial, with $k_{\perp}$ determined by the transverse geometry. The finite size beam reduction factor $\boldsymbol{R}$ enters through the boundary conditions at the radii $a$ and $b$ and is given in terms of Bessel functions by [Manickam et al., 1975]

$$
\begin{gathered}
R=\frac{\pi}{2}\left(k_{\perp} a\right) \frac{a}{b} \frac{Y_{0}\left(k_{\perp} b\right)}{Y_{1}\left(k_{\perp} b\right)}\left[J_{0}{ }^{2}\left(k_{\perp} a\right)+J_{1}{ }^{2}\left(k_{\perp} a\right)\right] \\
J_{0}\left(k_{\perp} b\right)=0
\end{gathered}
$$

The value $\omega_{b} R^{1 / 2} \cos \theta$ serves as a reduced effective beam plasma frequency. An important aspect of the dispersion (10) is the absorption of the transverse wavenumber $k_{\perp}$ and geometry effects into a single parameter $R$. Therefore from (11), $R$ is fixed when the mode number and the ratio $b / a$ is fixed. Figure 7 of Manickam et al. [1975] shows the values of $R$ as function of $b / a$ for the fundamental mode. For large $b / a \gg 1$, it has a logarithmic dependence approaching the value $R=0.1$.

In order to determine the conditions for absolute instability in our system we follow the techniques developed by Bers [1975], in the weak coupling approximation. For the beam waves the dispersion relation is

$$
D_{b}(k, \omega)=1-\frac{\omega_{b}^{2} R \cos ^{2} \theta}{\left(\omega-k_{z} V_{b}\right)^{2}}=0
$$

From this we find the usual fast and slow waves given by

$$
\omega-k_{z} V= \pm \omega_{b} R^{1 / 2} \cos \theta
$$

The slow wave is a negative energy wave while the fast is positive, i.e.,

$$
W_{b}= \pm \frac{2 \omega}{\omega_{b} R^{1 / 2} \cos \theta} \frac{1}{4} \varepsilon_{0}\left|\varepsilon_{b}\right|^{2}
$$

where $W_{b}$ is the wave energy, $\varepsilon_{b}$ the beam wave amplitude and $\varepsilon_{0}$ the free space dielectric constant. The negative energy wave can couple in synchronous interaction with the backward positive energy upper hybrid wave to produce an instability. Notice that for the upper hybrid wave

$$
D_{p}(k, \omega)=1-\frac{\omega_{0}^{2}\left(k_{z}\right)}{\omega^{2}}
$$

while the wave energy is

$$
W_{p}=\frac{2 \omega}{\omega_{0}\left(k_{z}\right)} \frac{1}{4} \varepsilon_{0}\left|\varepsilon_{p}\right|^{2}
$$

We examine now the situation where the slow wave of the beam interacts resonantly with the plasma wave $\omega_{0}$. We find a 
set of coupled equations

$$
\begin{aligned}
& \left(\frac{\partial}{\partial t}+v_{b} \frac{\partial}{\partial z}+v_{b}\right) U_{b}=C_{b p} U_{p} \\
& \left(\frac{\partial}{\partial t}+v_{p} \frac{\partial}{\partial z}+v_{p}\right) U_{p}=C_{p b} U_{b}
\end{aligned}
$$

where $v_{b}, v_{p}$ are the group velocities of the beam and plasma waves and $v_{b}, v_{p}$ are phenomenological damping coefficients of the two waves. $U_{p, b}$ are the usual normalized amplitudes defined as

$$
\left|U_{p, b}\right|^{2}=\frac{W_{p, b}}{\omega}=\left|\frac{\partial D_{p, b}}{\partial \omega}\right| \frac{\varepsilon_{0}}{4}\left|\varepsilon_{p, b}\right|^{2}
$$

[Bers, 1975; Davidson, 1972; Weiland and Wilhelmson, 1977] and the coupling coefficients $C_{b p}, C_{p b}$ are given by

$$
\begin{aligned}
& C_{b p}=\frac{-\frac{1}{4} \varepsilon_{b}^{*} J_{p b}}{W_{b}} \equiv \frac{P_{b p}}{W_{b}} \\
& C_{p b}=\frac{-\frac{1}{4} \varepsilon_{p}^{*} J_{b p}}{W_{p}} \equiv \frac{P_{p b}}{W_{p}}
\end{aligned}
$$

where $J_{p b}$ is the perturbed plasma current that interacts with the beam and $J_{b p}$ is the perturbed beam current that interacts with the plasma. For conservative interactions

$$
P_{b p}=-P_{p b}^{*}=-P_{p b}
$$

From (12)-(16) we find the growth rate

$$
\gamma=\frac{\left|P_{b p}\right|}{\left|W_{b} W_{p}\right|^{1 / 2}}=\frac{1}{2}\left(\frac{\omega_{b}^{2} R \cos ^{2} \theta}{\omega_{0}^{2}}\right)^{1 / 2} \omega_{0}
$$

Absolute instability requires $[$ Bers, 1975]

$$
\begin{gathered}
v_{b} v_{p}<0 \\
\gamma^{2}>v_{b} v_{p} \\
L>\frac{\left(\left|v_{b} v_{p}\right|^{1 / 2}\right)}{\gamma} \equiv L_{c}
\end{gathered}
$$

where $L$ is the system size in the $z$-direction. The first condition enters through the requirement that the unistable pulse encompasses the origin at all times. The second from the requirement that the pulse growth exceeds the dissipation. The last is equivalent to the breakdown length $L_{c}$ of an oscillator and implies that the feedback is stronger than convective losses. Notice that in the absence of wave reflecting boundaries only the upper hybrid branch can be absolutely unstable, since the lower hybrid branch corresponds to a forward wave (i.e., $v_{b} v_{p}>0$ ). In a plasma with $\omega_{e} / \Omega_{e}<1$, waves near the plasma frequency will be convectively unstable.

As mentioned in the introduction we associate the threshold of BPD in the Johnson chamber with an absolute instability near $\omega_{e}$. For our parameters the collisional ionization generates a plasma with $\omega_{e} / \Omega_{e}>2$, and the collisionality is such that condition $(19 b)$ is trivially satisfied. We therefore concentrate on (19c), for a backward wave in the upper hybrid range with $\omega_{e} / \Omega_{e} \gg 1$. The group velocity of the waves $v_{b}, v_{p}$ is

$$
\begin{gathered}
v_{b}=V_{b} \\
v_{p}=-2 V_{b} \frac{\Omega_{e}{ }^{2}}{\omega_{e}{ }^{2}} \cos ^{2} \theta \sin ^{2} \theta
\end{gathered}
$$

The value of $\sin \theta$ can be computed using (10e) and the first root of $(11 b)$, i.e., $k_{\perp} b=2.4$, giving

$$
\begin{aligned}
& \sin ^{2} \theta=\frac{1}{1+g^{2}} \\
& g^{2}=\frac{b^{2}}{(2.4)^{2}} \frac{\omega_{e}^{2}}{V_{b}^{2}}
\end{aligned}
$$

From (18), (19c), (20) and (21) we find the criterion for absolute instability as

$$
\omega_{b}^{2} \geq \frac{2(2)^{1 / 2}}{L}\left(\frac{n_{b}}{n_{0}}\right)^{1 / 2} \frac{\Omega_{e} V_{b}}{R^{1 / 2}} \sin \theta
$$

\section{BPD Ignition Scaling for Low Pressures}

Our conjecture associates the threshold for BPD triggering with the absolute instability criterion given by (22). This condition is a function of the beam and plasma parameters given by (9). From (9) and (22) we find the BPD triggering condition as

$$
I \geq I_{c} \equiv 1.2 \times 10^{-2} \frac{E_{b}^{3 / 2}}{p^{1 / 2}} \frac{D^{1 / 2}}{L} \frac{\left(\sin \theta \operatorname{sind} \theta_{d}\right)}{R^{1 / 2}}
$$

In order to make further progress we have to specify the value and the scaling of the diffusion coefficient $D$. We assume here that for the low pressure regime $p<p_{0}$ the diffusion is dominated by collective phenomena and obeys the Bohm diffusion law. Therefore

$$
D \simeq D_{B}=6 \times 10^{2}\left(\frac{T_{e}}{e V}\right) \frac{1}{B}
$$

The assumption of Bohm diffusion is consistent with the measured spectrum of low frequency fluctuations [Szuszczewicz et al., 1979] as well as the values of the diffusion they measured; for one set of parameters they measured values of $D_{B} \approx 2-9$ $\times 10^{2} \mathrm{~m}^{2} / \mathrm{s}$ which are much larger than the ones expected on the basis of classical collisions, and consistent with Bohm diffusion with $T_{e} \approx 0.5-1.5 \mathrm{eV}$. From (23) and (24) with $T_{e} \approx 0.5$ $\mathrm{eV}$ we find

$$
I_{c}=0.3 \frac{E_{b}^{3 / 2}}{p^{1 / 2}} \frac{1}{B^{1 / 2}} \frac{1}{L}\left(\frac{\operatorname{sub} \theta \sin \theta_{d}}{R^{1 / 2}}\right)
$$

For values of $g \leqslant 1$, the factor in parenthesis is of the order 3 , so that to within a factor of 2 the value of $I_{c}$ in amperes is

$$
I_{c}=\frac{E_{b}^{3 / 2}}{p^{1 / 2}} \frac{1}{B^{1 / 2}} \frac{1}{L}
$$

We proceed next to compare the predictions of $(25 b)$ with the experimental values. The most detailed case has been the one analyzed by Kellogg et al. [1982] for $E_{b}=1 \mathrm{keV}, p=5 \mu \mathrm{T}$, $L=20 \mathrm{~m}$ and $B=1 \mathrm{G}$ in which case $I_{c} \exp =20 \mathrm{~mA}$. For these values $(25 b)$ predicts $I_{c}=22 \mathrm{~mA}$. The minimum current for BPD triggering was $I_{c}{ }^{\text {exp }}=11 \mathrm{~mA}$ and occurred for $E_{b}=1$ $\operatorname{keV}, p=15 \mu \mathrm{T}, L=20 \mathrm{~m}$, and $B=1.14$. For these parameters (25b) gives $I_{c}=12 \mathrm{~mA}$. Table 1 compares the observed and predicted value of the threshold current in the ehamber for various experimental conditions. The agreement is rather satisfactory given the approximate nature of $(25 b)$ and the uncertainty in the experimental measurement of the pressure and $I_{c}$. We can, therefore, conclude that the above theory is consistent not only with the empirical scaling for the low pressure regime but, also reproduces with relative accuracy the numerical values of the critical current. It is interesting to compare the predictions of $(25 b)$ with the threshold observed 
TABLE 1. Comparison Between Theoretical Predictions and Experimental Results

\begin{tabular}{|c|c|c|c|c|c|}
\hline $\begin{array}{c}E_{b b}, \\
\mathrm{keV}\end{array}$ & $B, \mathbf{G}$ & $P, \mu \mathrm{T}$ & $\frac{I_{c}^{\text {exp }}}{\mathrm{mA}}$ & $\begin{array}{l}I_{c}{ }^{T} \\
\mathbf{m A}\end{array}$ & Comments \\
\hline 1.9 & 0.9 & $7-15$ & 34 & $35-40$ & \\
\hline 1.9 & 0.9 & 7 & 34 & 53 & \\
\hline 1.5 & 0.8 & 4 & 45 & 34 & \\
\hline 1.5 & 0.8 & 8 & 34 & 24 & \\
\hline 1.5 & 0.8 & 12 & 26 & 19 & \\
\hline 1.5 & 1.14 & 4 & 40 & 28 & \\
\hline 1.5 & 1.14 & 7 & 22 & 32 & large \\
\hline 1.5 & 1.14 & 15 & 12 & 22 & chamber \\
\hline 1.5 & 1.5 & 4 & 30 & 25 & results \\
\hline 1.5 & 1.5 & 2 & 50 & 35 & $L=20 \mathrm{~m}$ \\
\hline 1.3 & 1.5 & $6-12$ & 12 & $17-24$ & \\
\hline 1.3 & 0.9 & $7-15$ & 28 & $20-30$ & \\
\hline 1 & 1.5 & 4 & 28 & 23 & \\
\hline 1 & 1.14 & 15 & 11 & 12 & \\
\hline 1 & 1.0 & 5 & 20 & 22 & \\
\hline 0.8 & 0.9 & $8-15$ & 7.8 & $9-13$ & \\
\hline 0.75 & 1.5 & 2 & 32 & 19 & \\
\hline 1.6 & 38 & 100 & 8 & 12 & \\
\hline 1.6 & 38 & 71 & 11 & 15 & small \\
\hline 1.6 & 38 & 50 & 26 & 18 & chamber \\
\hline 1.6 & 20 & 100 & 10 & 17 & results \\
\hline 1.6 & 11.5 & 100 & 20 & 23 & $L=2.6 \mathrm{~m}$ \\
\hline 1.6 & 7.7 & 100 & 28 & 28 & \\
\hline 1.6 & 5.5 & 100 & 40 & 33 & \\
\hline
\end{tabular}

in a comparable experiment by Cabral et al. [1969]. For $E_{b}=$ $1.5 \mathrm{keV}, P=5 \times 10^{-4}, B=325 \mathrm{G}$ and $L=.45 \mathrm{~m}$, the observed threshold current was $I_{c}{ }^{\exp }=16 \mathrm{~mA}$ while (25b) will predict $I_{\mathrm{c}} \approx 9 \mathrm{~mA}$. We should also mention that $(25 b)$ was a good fit to the small chamber results of Konradi et al. [1983].

An approximate criterion for the ignition of BPD was given before [Rowland et al., 1981; Papadopoulos, 1981] and was found consistent with the observations at the J.S.F.C. tank [Szuszczewicz et al., 1982] as $\omega_{e} / \boldsymbol{\Omega}_{e}>5$. It is appropriate to comment on its relationship to the present more detailed considerations. Referring to (23) we note that $I_{c} \sim \sin \theta$ so that for $\sin \theta \ll 1, I_{c}$ becomes very small, independently of other considerations. From (21) $\sin \theta \ll 1$ corresponds to

$$
g=\frac{b}{2.4} \frac{\omega_{e}}{V_{b}} \gg 1
$$

which is the opposite limit from the one considered before. Taking $b \approx O(a)$ and using $(1 a)$ we recover the condition $\omega_{e} / \Omega_{e}>2.4 /\left(\sin \theta_{d}\right) \approx 5$ as an approximate condition at which absolute instability develops. This criterion is extremely relevant for cases with preionized plasma such as the ionosphere at daylight conditions or for high altitude injection (i.e., $F$ peak). The $\omega_{e} / \Omega_{e}>5$ criterion is a sufficient but not a necessary condition, and accounts in a natural fashion for the observed hysterysis during BPD extinction [Bernstein et al., 1979].

Before closing this section we should comment briefly on the scaling for the high pressure regime. If we assume that similar physical considerations, i.e., absolute instability at $\omega_{e}$, determine the value of $I_{c}$ in this regime also, then a change in the scaling would imply that the higher collisionality changes the scaling of $D$ or the scaling of the growth rate $\gamma$ or both. The absence of detailed information in this regime makes a quantitative theoretical study impossible. We mention bere only that Papadopoulos [1984] conjectured that the transition is caused by transition of the diffusion in the high pressure regime from Bohm $\left(D_{B}\right)$ to classical $\left(D_{c}\right)$. The ratio $D_{c} / D_{B} \sim$ $\left(T_{e}^{1 / 2} p\right) / B$. Therefore instead of the scaling given by (25) we find

$$
I_{c} \sim \frac{E_{b}^{3 / 2}}{B L} T_{e}^{1 / 4}
$$

Namely the critical current scales as $1 / B$ rather than $1 / B^{1 / 2}$ and $I_{c}$ is independent of the pressure instead of $1 / p^{1 / 2}$. In order for $I_{c}$ to increase monotonically with pressure $T_{e} \sim p$. Whether such a relationship is justified depends on the scalings of the heating rates and the heat conduction processes. Such a study will be justified after more detailed data become available.

\section{Summary and Conclusions}

We have presented a detailed physical analysis of BPD threshold scaling based on the conjecture proposed by Papadopoulos [1982], that the BPD threshold is associated with the triggering of an absolute instability near $\omega_{e}$. This conjecture predicts a scaling

$$
I_{c} \sim \frac{E_{b}^{3 / 2}}{p^{1 / 2} B^{1 / 2} L}
$$

The predicted scaling and the numerical values of $I_{c}$, as well as the dominance of Bohm diffusion in the low pressure regime are in agreement with the data from JSC tank experiment [Bernstein et al., 1979; Szuszczewicz et al., 1979]. It is important to restate some of the key assumptions of the theory and their consequences, since many were inspired from the JSC tank parameters and might not be applicable to other situations.

1. Weak magnetic field in the sense that at prebreakdown $\omega_{e} / \Omega_{e} \geq 2$. For situations where $\omega_{e} / \Omega_{e}<1$, the $\omega_{e}$ waves lie in the lower hybrid branch of the dispersion curve which corresponds to forward waves and therefore produces convective instability. The expected scaling should be derived from different considerations. The case $\Omega_{e}<\omega_{e}<2 \Omega_{e}$, requires special consideration due to the presence of strong cyclotron damping which was neglected here. Note also that end plate reflections can produce an absolute instability even in the lower hybrid branch.

2. Thin beam in the sense $a \ll b$. For situations where $a \approx b$, axial losses could be dominant and the more general (8) and (9) should be used instead of (7).

3. Weak coupling limit (14) are valid in the limit where the parameter $\left(\omega_{b} / \omega_{e}\right)^{1 / 2} \approx\left(n_{b} / n_{0}\right)^{1 / 2} \ll 1$. Otherwise the saddle point method [Le Queau et al., 1980] should be used to determine the threshold condition given by (22).

4. In associating the threshold for absolute instability with the BPD ignition we implicitly assumed that the energy deposition from the beam to the plasma will produce ambient electron fluxes whose ionization rate exceeds the beam ionization rate. This seems to be clearly satisfied in the low pressure regime. However as noted in Papadopoulos et al. [1983] by increasing the pressure there is a limit at which the energy deposition by the beam plasma instability is not sufficient to overcome line emission so that the electron energy stays below the ionization energy. This case will have many of the BPD signatures (i.e., broadening of the irradiated region) but the electron plasma density will be controlled by beam ionization only (i.e., BPD without D). The small chamber experiment [Bernstein et al., 1983] is possibly indicative of such behavior.

Before closing we should comment on the applicability of the above concepts to electron beam injection in space. There are two fundamental differences between the laboratory and the ionospheric beam injection: (1) the existence of ambient plasma with long density gradient scales (i.e., $L \rightarrow \infty$ ) in the 
ionosphere; (2) the laboratory experiments are steady state, while the vehicle motion across the magnetic field line can be thought as producing beam pulses with time length $\tau=U / a$ where $U$ is the cross-field motion of the vehicle (i.e., $1-2 \mathrm{~km} / \mathrm{s}$ for rockets, $4-8 \mathrm{~km} / \mathrm{s}$ for the shuttle).

In assessing BPD for the ionospheric case we have to ask the following questions:

1. Is the plasma density produced by the collisional ionization due to the beam during $t \ll \tau$, larger than the ambient ionospheric density?

2. Is there a beam instability at $\omega_{e}$ based on the ambient ionospheric plasma density?

3. Is the ionization time due to the hot electrons produced by the instability shorter than the injection time $\tau$ (i.e., $v_{\text {ion }}$ $\tau \gg 1)$ ?

If the answer to question (1) is yes and to question (2) no, the threshold condition is similar to (25), with the length $L$ given by the plasma density gradient due to collisional ionization by the beam. In view of the short injection time, however, (25) is sufficient only for the beam plasma instability (BPI) but not BPD. In order to have BPD we need in addition to (25) a positive answer to question be given by (22) with $n_{0}$ the value of the ambient plasma density. BPD requires in addition $v_{\text {ion }}$ $\tau>1$. The above comments should be only taken as guidelines. More precise considerations require nonlinear BPI computations for the evaluation of $v_{\text {ion }}$ as a function of the beam parameters and will be reported in the future.

During the refereeing period, Llobet et al. [1985] confirmed the above results in a detailed study in the small chamber.

Acknowledgments. This work has evolved from many years of association with the team performing the JSC experiments (H. Anderson, B. Bernstein, J. Jost, T. Hallinan, P. Kellogg, H. Leinbach and E. Szuszczewicz) whose contribution to this work cannot be overemphasized. Special thanks belong to B. Bernstein for introducing me to the subject of BPD and for many heated but stimulating discussions as well as to my collaborator in previous BPD work, $L$. Linson. Sincere thanks to one of the referees for pointing out an inconsistency in the derived high pressure scaling in an earlier version of the manuscript. The work was supported in part by NASA and in part by ONR N00014-79-C-0665.

The Editor thanks L. D. Smullin and T. Hallinan for their assistance in evaluating this paper.

\section{REFERENCES}

Bernstein, W., H. Leinbach, P. Kellogg, S. Monson, T. Hallinan, $O$ K. Garriott, A. Konradi, J. McCoy, P. Daly, B. Baker, and H. R. Anderson, Electron beam injection experiments: The beam-plasmadischarge at low pressures and magnetic field strengths, Geophys. Res. Lett., 5, 127, 1978.

Bernstein, W., H. Leinbach, P. J. Kellogg, S. J. Monson, and T. Hallinan, Further laboratory measurements of the beam-plasma discharge, J. Geophys. Res., 84, 7271, 1979.

Bernstein, W., B. A. Whalen, F. R. Harris, A. G. McNamara, and A. Konradi, Laboratory studies of the charge neutralization of a rocket payload during electron beam emission, Geophys. Res. Lett., $7,93,1980$.

Bernstein, W., J. O. McGarity, and A. Konradi, Electron beam injection experiments: replication of flight observations in a laboratory beam plasma discharge, Geophys. Res. Lett., 10, 1124, 1983.

Bers, A., Linear waves and instabilities, in Plasma Physics, Les Houches 1972, edited by C. DeWitt and J. Peyraud, Gordon and Breach, New York, p. 113, 1975.

Briggs, R. J., Electron-Stream Interactions With Plasma, MIT Press, Cambridge, Mass., 1964.

Cabral, J. A., H. J. Hoffman, and J. A. van Wakeren, Influence of the magnetic field in the interaction between a monoenergetic beam and its own created plasma, in Phenomena in lonized Gases, edited by G. Musa, I. Ghica, A. Popescu, and L. Nastase, Institute of Physics, Academy of Romani, Bucharest, 1969.

Davidson, R. C., Methods in Non-Linear Plasma Theory, chap. 6, Academic, Orlando, Fla., 1972.

Galeev, A. A., E. V. Mishin, R. Z. Sagdeev, V. D. Shapiro, and V. I.
Shevchenko, Discharge in the region around a rocket following the injection of electron beams in the ionosphere, Sov. Phys. Dokl., 21, $641,1976$.

Galeev, A. A., R. Z. Sagdeev, V. D. Shapiro, and V. I. Shevchenko, Beam plasma discharge and suprathermal electron tails, Active Experiments in Space, Eur. Space Agency Spec. Publ., ESA-SP-195, $151,1983$.

Getty, W. D., and L. D. Smullin, Beam-plasma discharge: Buildup of oscillations, J. Appl. Phys., 34, 3421, 1963.

Kawashima, N., S. Sasaki, K. Takahashi, and T. Obayashi, BPD experiments in the laboratory, Active Experiments in Space, Eur. Space Agency Spec. Publ., ESA-SP-195, 181, 1983.

Kellogg, P. J., H. R. Anderson, W. Bernstein, T. J. Hallinau, R. H. Holzworth, R. J. Jost, H. Leinbach, and E. P. Szuszczewicz, Laboratory simulation of injection particle beams in the ionosphere, in Proceedings of NATO Advanced Research Institute on Artificial Particle Beams in Space Plasma Physics, edited by B. Grandall, p. 289, Plenum, New York, 1982.

Kharchenko, I. F., Va. B. Feinberg, R. M. Nikolayev, E. A. Kornilov, E. Lutsenko, and N. S. Pedenko, The interaction of an electron beam with a plasma in a magnetic field, Sov. Phys. Tech. Phys., 6, $551,1962$.

Konradi, A., W. Bernstein, D. L. Bulgher, J. O. Mc Garrity, and J. L. Winkler, Initial results from a laboratory size beam plasma discharge, Active Experiments in Space, Eur. Space Agency. Spec. Publ., ESA-SP-195, 185, 1983.

Le Queau, D., R. Pellat, and A. Saint-Marc, An investigation of electrostatic linear instabilities of a radially limited electron beam, $A n n$. Geophys., 36, 423, 1980.

Linson, L. M., and K. Papadopoulos, Review of the status of theory and experiment for injection of energetic electron beams in space, Rep. PAPS-69/SA1-D23-459-LJ, Sci. Appl., Inc., La Jolla Calif., April 1980.

Llobet, X., W. Bernstein, and A. Konradi, The spatial evolution of energetic electrons and plasma waves during the steady state beam plasma discharge, J. Geophys. Res., 90, 5187, 1985.

Lyakhov, S. B., A. D. Maiorov, G. G. Managadze, O. A. Povalyev, A. I. Chmil, L. Yu Kochmarev, E. G. Shustin, M. F. Friedrich, W. K. Riedler, A. N. Lalishvili, A. Kiraga, Z. Kloss, Z. Kravchic, and N. A. Leonov, Laboratory studies for beam plasma discharge in experiments in rockets, Planet. Space Res., 30, 347, 1982.

Manickam, J., W. Carr, B. Rosen, and M. Seidl, Convective and absolute instabilities in beam plasma systems, Phys. Fluids, 18, 369, 1975.

Papadopoulos, K., Non-linear stabilization of beam plasma instabilities by parametric effects, Phys. Fluids, 18, 1769, 1975.

Papadopoulos, K., Theory of beam plasma discharge, in Proceedings of NATO Advanced Research Institute on Artificial Particle Beams in Space Plasma Physics, edited by B. Grandal, p. 505, Plenum, New York, 1982.

Papadopoulos, K., On the plasma physics of the beam plasma discharge, Comments in Plasma Phys. Contr. Fusion, 9, 1, 1985.

Papadopoulos, K., and T. Coffey, Nonthermal features of the auroral plasma due to the precipitating electrons, J. Geophys. Res., 79, 674, 1974.

Papadopoulos, K., and H. L. Rowland, Collisionless effects on the spectrum of secondary auroral electrons at low altitudes, $J$. Geophys. Res., 83, 5768, 1978.

Papadopoulos, K., C. L. Chang, and K. Ko, Collective plasma effects on electron beam injection from rockets, Rep. SAI 83-1215, Sci. Appl. Inc., McLean, Va., 1983.

Rowland, H. L., C. L. Change, and K. Papadopoulos, Scaling of the beam-plasma discharge, J. Geophys. Res., 86, 9215, 1981.

Szuszczewicz, E. P., D. N. Walker, J. C. Holmes, and H. Leinbach, Plasma diffusion in a space simulation beam plasma discharge, Geophys. Res. Lett., 6, 201, 1979.

Szuszczewicz, E. P., K. Papadopoulos, W. Bernstein, C. S. Liu, and D. N. Walker, Threshold criterion for a beam plasma discharge, $J$. Geophys. Res., 87, 1565, 1982.

Weiland, J., and H. Wilhelmson, Coherent Non-Linear Interaction of Waves in Plasmas, Pergamon, New York, 1977.

K. Papadopoulos, Department of Physics and Astronomy, University of Maryland, College Park, MD 20742.

(Received February 8, 1984;

revised July 22, 1985;

accepted November 11, 1985.) 